\title{
Periodic solutions of second-order nonlinear system of integro-differential equations unsolvable for the highest derivative
}

\author{
R. N. Butris \\ Department of Mathematics \\ College of Education/ University of Mosul \\ Received \\ Accepted \\ 21/11/2006 \\ 21/02/2007
}

\section{الملغص}

يتضمن البهث درلمة وجود وقريب الحلول الدوري ة ل ـبعض أظمه المة المع ـادلات

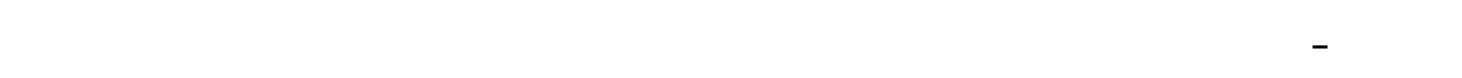

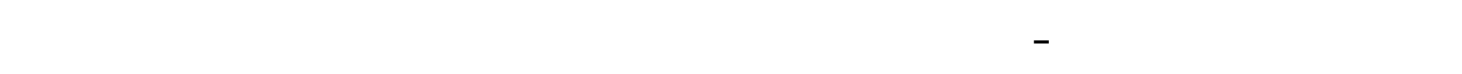
ـ ـ A. M. Samoilenko. وكذالك تؤدي هذه الدرلسة اللى تحسين وتوسيع الطاريقة أعلاه.

\section{ABSTRACT}

In this paper we study the existence and approximation of the periodic solutions for certain systems of second-order of integrodifferential equations unsolvable for the highest derivative by using the numerical analytic method for ordinary differential equations of A. M. Samoilenko which given. Also these investigation lead us to the improving the extending the above method.

\section{Introduction}

Consider the following system of second-order integro-differential equation, which has the form:

$$
\frac{d^{2} x}{d t^{2}}=f\left(t, x, \dot{x}, \ddot{x}, \int_{t-T}^{t} g(s, x(s), \dot{x}(s), \ddot{x}(s)) d s\right)
$$

where $x \in D \subseteq R^{n}, D$ is a closed and bounded domain.

The vector functions $f(t, x, \dot{x}, \ddot{x}, w)$ and $g(t, x, \dot{x}, \ddot{x})$ are defined on the domain:

$$
\begin{aligned}
(t, x, \dot{x}, \ddot{x}, w) & \in R^{1} \times D \times D_{1} \times D_{2} \times D_{3} \\
& =(-\infty, \infty) \times D \times D_{1} \times D_{2} \times D_{3}
\end{aligned}
$$

which are continuous in $(t, x, \dot{x}, \ddot{x}, w)$ and periodic in t of period $\mathrm{T}$, where $D_{1}, D_{2}$ and $D_{3}$ are bounded domains subset of Euclidean spaces $R^{n}$ and $R^{m}$ respectively. 
Suppose that the vector functions $f(t, x, \dot{x}, \ddot{x}, w)$ and $g(t, x, \dot{x}, \ddot{x})$ are satisfy the following inequalities:

$$
\begin{aligned}
& \|f(t, x, \dot{x}, \ddot{x}, w)\| \leq M \quad, \quad\|g(t, x, \dot{x}, \ddot{x})\| \leq M \quad, \\
& \left\|f\left(t, x_{1}, \dot{x}_{1}, \ddot{x}_{1}, w_{1}\right)-f\left(t, x_{2}, \dot{x}_{2}, \ddot{x}_{2}, w_{2}\right)\right\| \leq K_{1}\left\|x_{1}-x_{2}\right\|+K_{2}\left\|\dot{x}_{1}-\dot{x}_{2}\right\|+ \\
& +K_{3}\left\|\ddot{x}_{1}-\ddot{x}_{2}\right\|+K_{4}\left\|w_{1}-w_{2}\right\|, \\
& \left\|g\left(t, x_{1}, \dot{x}_{1}, \ddot{x}_{1}\right)-g\left(t, x_{2}, \dot{x}_{2}, \ddot{x}_{2}\right)\right\| \leq L_{1}\left\|x_{1}-x_{2}\right\|+L_{2}\left\|\dot{x}_{1}-\dot{x}_{2}\right\|+L_{3}\left\|\ddot{x}_{1}-\ddot{x}_{2}\right\|,
\end{aligned}
$$

for all $t \in R^{1}$ and $x, x_{1}, x_{2} \in D, \dot{x}, \dot{x}_{1}, \dot{x}_{2} \in D_{1} \quad, \quad \ddot{x}, \ddot{x}_{1}, \ddot{x}_{2} \in D_{2}$ and $w, w_{1}, w_{2} \in D_{3}$, where $M, K_{1}, K_{2}, K_{3}, K_{4}$ and $L_{1}, L_{2}, L_{3}$ are a positive constants , $\|\|=.\max _{0 \leq t \leq T}|\cdot|$.

We define the non-empty sets as follows:

$$
\begin{aligned}
& D_{N}=D-N, \\
& D_{1 N_{1}}=D_{1}-N_{1}, \\
& D_{2 N_{2}}=D_{2}-N_{2}, \\
& D_{3 N_{3}}=D_{3}-N_{3}
\end{aligned},
$$

where $\quad N=\frac{M T^{2}}{6} \quad, \quad N_{1}=\frac{5 M T}{6} \quad, \quad N_{2}=2 M$

$$
N_{3}=\left(\frac{M T^{2}}{6} L_{1}+\frac{5 T M}{6} L_{2}+2 M L_{3}\right) T+M T \text {. }
$$

Furthermore, we suppose that the largest eigen-value $q_{\max }$ of the following matrix:

$$
Q_{0}=\left(\begin{array}{ccc}
\frac{T^{2}}{6}\left(K_{1}+K_{4} L_{1} T\right) & \frac{T^{2}}{6}\left(K_{2}+K_{4} L_{1} T\right) & \frac{T^{2}}{6}\left(K_{3}+K_{4} L_{1} T\right) \\
\frac{5 T}{6}\left(K_{1}+K_{4} L_{2} T\right) & \frac{5 T}{6}\left(K_{2}+K_{4} L_{2} T\right) & \frac{5 T}{6}\left(K_{3}+K_{4} L_{2} T\right) \\
2\left(K_{1}+K_{4} L_{3} T\right) & 2\left(K_{2}+K_{4} L_{3} T\right) & 2\left(K_{3}+K_{4} L_{3} T\right)
\end{array}\right)
$$

is less than one, i.e.

$$
\left[\frac{T^{2}}{6}\left(K_{1}+K_{4} L_{1} T\right)+\frac{5 T}{6}\left(K_{2}+K_{4} L_{2} T\right)+2\left(K_{3}+K_{4} L_{3} T\right)\right]<1
$$




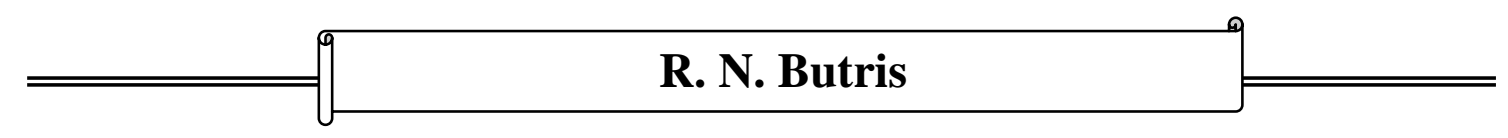

\section{Lemma 1 :}

Let $f(t)$ be a continuous vector function in the interval $[0, T]$, then:

$\left\|\int_{0}^{t}\left(f(t)-\frac{1}{T} \int_{0}^{T} f(s) d s\right) d s\right\| \leq \alpha(t) M$,

where $M=\max _{t \in[0, T]}\|f(t)\| \quad$ and $\quad \alpha(t)=2 t\left(1-\frac{t}{T}\right)$.

For the proof see [2].

We define an operator $\mathrm{L}$ as follows:

$(L f)(t)=L f(t)=\int_{0}^{t}\left(f(t)-\frac{1}{T} \int_{0}^{T} f(s) d s\right) d s$

we obtain

$L(L f)(t)=L^{2} f(t)=\int_{0}^{t}\left(L f(t)-\frac{1}{T} \int_{0}^{T} L f(s) d s\right) d s$.

It is obvious that if $f(t)$ is continuous on the interval $[0, T]$, then $L f(t)$ and $L^{2} f(t)$ are also continuous on the same interval.

By lemma 1, we get:

$\|L f(t)\| \leq \alpha(t) \max _{t \in[0, T]}\|f(t)\|$

and

$\left\|L^{2} f(t)\right\| \leq \alpha(t)\|L f(t)\| \leq \frac{T}{3} \alpha(t) M \leq \frac{T^{2}}{6} M$

for all $t \in[0, T]$ and $\alpha(t) \leq \frac{T}{2}$.

\section{Approximation Solution of (1)}

The investigation of approximation solution of the system (1) will be introduced by the following theorem.

\section{Theorem 1:}

If the system of second-order integro-differential equations (1) satisfy the inequalities (3), (4) with assumptions (5), (6) and the condition (8) has a periodic solution $x=x\left(t, x_{0}\right)$, passing through the point $\left(0, x_{0}\right)$, $x_{0} \in D_{f}$, then the sequence of functions: 
$x_{m+1}\left(t, x_{0}\right)=x_{0}+L^{2} f\left(t, x_{m}\left(t, x_{0}\right), \dot{x}_{m}\left(t, x_{0}\right), \ddot{x}_{m}\left(t, x_{0}\right), \int_{t-T}^{t} g\left(s, x_{m}\left(s, x_{0}\right), \dot{x}_{m}\left(s, x_{0}\right), \ddot{x}_{m}\left(s, x_{0}\right)\right) d s\right)$

with

$x_{0}\left(t, x_{0}\right)=x_{0} \quad, \quad \frac{d x_{m}\left(t, x_{0}\right)}{d t}=\dot{x}_{m}\left(t, x_{0}\right) \quad, \quad \frac{d^{2} x_{m}\left(t, x_{0}\right)}{d t^{2}}=\ddot{x}_{m}\left(t, x_{0}\right)$,

$\mathrm{m}=0,1,2, \ldots$,

is periodic in $t$ of period $\mathrm{T}$, and is uniformly convergent as $m \rightarrow \infty$ in the domain:

$\left(t, x_{0}\right) \in R^{1} \times D_{f}=(-\infty, \infty) \times D_{f}$,

to the function $x_{\infty}\left(t, x_{0}\right)$ defined in the domain (10), which is periodic in $\mathrm{t}$ of period $\mathrm{T}$ and satisfying the system of integral equations:

$x\left(t, x_{0}\right)=x_{0}+L^{2} f\left(t, x\left(t, x_{0}\right), \dot{x}\left(t, x_{0}\right), \ddot{x}\left(t, x_{0}\right), \int_{t-T}^{t} g\left(s, x\left(s, x_{0}\right), \dot{x}\left(s, x_{0}\right), \ddot{x}\left(s, x_{0}\right)\right) d s\right)$

which is a unique solution of the system (1) provided that:

$\left(\begin{array}{l}\left\|x_{\infty}-x_{m}\right\| \\ \left\|\dot{x}_{\infty}-\dot{x}_{m}\right\| \\ \left\|\ddot{x}_{\infty}-\ddot{x}_{m}\right\|\end{array}\right) \leq Q_{0}^{m}(E-Q)^{-1} v_{0}$

where $v_{0}=\left(\begin{array}{c}N_{1} \\ N_{2} \\ N_{3}\end{array}\right)$ and $\mathrm{E}$ is identity matrix.

\section{Proof:}

Setting $\mathrm{m}=0$ and using (9), we get

$$
\begin{aligned}
\left\|x_{1}\left(t, x_{0}\right)-x_{0}\right\| & =\left\|L^{2} f\left(t, x_{0}, 0,0, \int_{t-T}^{t} g\left(s, x_{0}, 0,0\right) d s\right)\right\| \\
\leq & \left(1-\frac{t}{T}\right) \int_{0}^{t}\left\|L f\left(s, x_{0}, 0,0, \int_{s-T}^{s} g\left(\tau, x_{0}, 0,0\right) d \tau\right)\right\| d s+ \\
& +\frac{t}{T} \int_{t}^{T}\left\|L f\left(s, x_{0}, 0,0, \int_{s-T}^{s} g\left(\tau, x_{0}, 0,0\right) d \tau\right)\right\| d s \leq M \frac{T^{2}}{6}
\end{aligned}
$$

Hence

$$
\left\|x_{1}\left(t, x_{0}\right)-x_{0}\right\| \leq M \frac{T^{2}}{6}
$$




\section{R. N. Butris}

So that $x_{1}\left(t, x_{0}\right) \in D$ for all $t \in R^{1}$ and $x_{0} \in D_{N}$. Moreover on differentiating $x_{1}\left(t, x_{0}\right)$, we find $\dot{x}_{1}\left(t, x_{0}\right)=L f\left(t, x_{0}, 0,0, \int_{t-T}^{t} g\left(s, x_{0}, 0,0\right) d s\right)-\frac{1}{T} \int_{0}^{T} L f\left(t, x_{0}, 0,0, \int_{t-T}^{t} g\left(s, x_{0}, 0,0\right) d s\right) d t$ and hence

$$
\begin{aligned}
\left\|\dot{x}_{1}\left(t, x_{0}\right)\right\| & \leq\left\|f\left(t, x_{0}, 0,0, \int_{t-T}^{t} g\left(s, x_{0}, 0,0\right) d s\right)\right\|-\frac{1}{T} \int_{0}^{T}\left\|L f\left(t, x_{0}, 0,0, \int_{t-T}^{t} g\left(s, x_{0}, 0,0\right) d s\right)\right\| d t \\
& \leq \alpha(t) M+\frac{1}{T} \int_{0}^{T} \alpha(t) M d t \\
& \leq\left(\alpha(t)+\frac{T}{3}\right) M \leq \frac{5 T}{6} M
\end{aligned}
$$

From (4) and (5), we get $\dot{x}_{1}\left(t, x_{0}\right) \in D_{1}$ for $x_{0} \in D_{N}$ and $t \in R^{1}$, also on differentiating $\dot{x}_{1}\left(t, x_{0}\right)$ we find $\ddot{x}_{1}\left(t, x_{0}\right)=f\left(t, x_{0}, 0,0, \int_{t-T}^{t} g\left(s, x_{0}, 0,0\right) d s\right)-\frac{1}{T} \int_{0}^{T} f\left(t, x_{0}, 0,0, \int_{t-T}^{t} g\left(s, x_{0}, 0,0\right) d s\right) d t$

Therefore

$$
\begin{aligned}
\left\|\ddot{X}_{1}\left(t, x_{0}\right)\right\| & \leq\left\|f\left(t, x_{0}, 0,0, \int_{t-T}^{t} g\left(s, x_{0}, 0,0\right) d s\right)\right\|+\frac{1}{T} \int_{0}^{T}\left\|f\left(t, x_{0}, 0,0, \int_{t-T}^{t} g\left(s, x_{0}, 0,0\right) d s\right)\right\| d t \\
& \leq 2 M
\end{aligned}
$$

From (6) and (15), we have $\ddot{x}_{1}\left(t, x_{0}\right) \in D_{2}$ for all $x_{0} \in D_{N}$ and $t \in R^{1}$.

Using the inequalities (4), (13), (14) and (15), we find

$$
\begin{aligned}
\left\|\int_{t-T}^{t}\left(g\left(s, x_{1}\left(s, x_{0}\right), \dot{x}_{1}\left(s, x_{0}\right), \ddot{x}_{1}\left(s, x_{0}\right)\right)-g\left(s, x_{0}, 0,0\right)+g\left(s, x_{0}, 0,0\right)\right) d s\right\| \leq \\
\quad \leq \int_{t-T}^{t}\left\|g\left(s, x_{1}\left(s, x_{0}\right), \dot{x}_{1}\left(s, x_{0}\right), \ddot{x}_{1}\left(s, x_{0}\right)\right)-g\left(s, x_{0}, 0,0\right)\right\| d s+M T \\
\quad \leq \int_{t-T}^{T}\left(L_{1}\left\|x_{1}\left(s, x_{0}\right)-x_{0}\right\|+L_{2}\left\|\dot{x}_{1}\left(s, x_{0}\right)\right\|+L_{3}\left\|\ddot{x}_{1}\left(s, x_{0}\right)\right\| d s+M T\right. \\
\quad \leq\left(\frac{M T^{2}}{6} L_{1}+\frac{5 T M}{6} L_{2}+2 M L_{3}\right) T+M T=N_{3}
\end{aligned}
$$

From (6) and (16), we get $w_{1}(t) \in D_{3}$ for all $t \in R^{1}$ and $x_{0} \in D_{N}$. 
Thus by induction we can prove that $x_{m}\left(t, x_{0}\right) \in D, \dot{x}_{m}\left(t, x_{0}\right) \in D_{1}$, $\ddot{x}_{m}\left(t, x_{0}\right) \in D_{2}$ and $w_{m}(t) \in D_{3}$, for $x_{0} \in D_{N}, \mathrm{~m}=1,2,3, \ldots$.

We claim that the sequence of functions $x_{m}\left(t, x_{0}\right)$ is uniformly convergent on the domain (10).

By using (9) and (13) the following inequalities are holds:

$$
\begin{aligned}
&\left\|x_{m+1}\left(t, x_{0}\right)-x_{m}\left(t, x_{0}\right)\right\| \leq \alpha(t) \frac{T}{3}[\left(K_{1}+K_{4} L_{1} T\right)\left\|x_{m}\left(t, x_{0}\right)-x_{m-1}\left(t, x_{0}\right)\right\|+ \\
&+\left(K_{2}+K_{4} L_{2} T\right)\left\|\dot{x}_{m}\left(t, x_{0}\right)-\dot{x}_{m-1}\left(t, x_{0}\right)\right\|+ \\
&\left.+\left(K_{3}+K_{4} L_{3} T\right)\left\|\ddot{x}_{m}\left(t, x_{0}\right)-\ddot{x}_{m-1}\left(t, x_{0}\right)\right\|\right], \\
& \ldots \ldots(17) \ldots \\
&\left\|\dot{x}_{m+1}\left(t, x_{0}\right)-\dot{x}_{m}\left(t, x_{0}\right)\right\| \leq\left(\alpha(t)+\frac{T}{3}\right)\left[\left(K_{1}+K_{4} L_{1} T\right)\left\|x_{m}\left(t, x_{0}\right)-x_{m-1}\left(t, x_{0}\right)\right\|+\right. \\
&+\left(K_{2}+K_{4} L_{2} T\right)\left\|\dot{x}_{m}\left(t, x_{0}\right)-\dot{x}_{m-1}\left(t, x_{0}\right)\right\|+ \\
&\left.+\left(K_{3}+K_{4} L_{3} T\right)\left\|\ddot{x}_{m}\left(t, x_{0}\right)-\ddot{x}_{m-1}\left(t, x_{0}\right)\right\|\right],
\end{aligned}
$$

and

$$
\begin{aligned}
\left\|\ddot{x}_{m+1}\left(t, x_{0}\right)-\ddot{x}_{m}\left(t, x_{0}\right)\right\| \leq 2\left[\left(K_{1}\right.\right. & \left.+K_{4} L_{1} T\right)\left\|x_{m}\left(t, x_{0}\right)-x_{m-1}\left(t, x_{0}\right)\right\|+ \\
& +\left(K_{2}+K_{4} L_{2} T\right)\left\|\dot{x}_{m}\left(t, x_{0}\right)-\dot{x}_{m-1}\left(t, x_{0}\right)\right\|+ \\
& \left.+\left(K_{3}+K_{4} L_{3} T\right)\left\|\ddot{x}_{m}\left(t, x_{0}\right)-\ddot{x}_{m-1}\left(t, x_{0}\right)\right\|\right] .
\end{aligned}
$$

Rewrite the inequalities (17), (18) and (19) in vector form as: $v_{m+1}(t) \leq Q(t) v_{m}(t)$,

where

$$
\begin{aligned}
& v_{m+1}(t)=\left(\begin{array}{l}
\left\|x_{m+1}\left(t, x_{0}\right)-x_{m}\left(t, x_{0}\right)\right\| \\
\left\|\dot{x}_{m+1}\left(t, x_{0}\right)-\dot{x}_{m}\left(t, x_{0}\right)\right\| \\
\left\|\ddot{x}_{m+1}\left(t, x_{0}\right)-\ddot{x}_{m}\left(t, x_{0}\right)\right\|
\end{array}\right), \\
& Q(t)=\left(\begin{array}{ccc}
\alpha(t) \frac{T}{3}\left(K_{1}+K_{4} L_{1} T\right) & \alpha(t) \frac{T}{3}\left(K_{2}+K_{4} L_{1} T\right) & \alpha(t) \frac{T}{3}\left(K_{3}+K_{4} L_{1} T\right) \\
\left(\alpha(t)+\frac{T}{3}\right)\left(K_{1}+K_{4} L_{2} T\right) & \left(\alpha(t)+\frac{T}{3}\right)\left(K_{2}+K_{4} L_{2} T\right) & \left(\alpha(t)+\frac{T}{3}\right)\left(K_{3}+K_{4} L_{2} T\right) \\
2\left(K_{1}+K_{4} L_{3} T\right) & 2\left(K_{2}+K_{4} L_{3} T\right) & 2\left(K_{3}+K_{4} L_{3} T\right)
\end{array}\right), \\
& v_{m}(t)=\left(\begin{array}{l}
\left\|x_{m}\left(t, x_{0}\right)-x_{m-1}\left(t, x_{0}\right)\right\| \\
\left\|\dot{x}_{m}\left(t, x_{0}\right)-\dot{x}_{m-1}\left(t, x_{0}\right)\right\| \\
\left\|\ddot{x}_{m}\left(t, x_{0}\right)-\ddot{x}_{m-1}\left(t, x_{0}\right)\right\|
\end{array}\right) .
\end{aligned}
$$




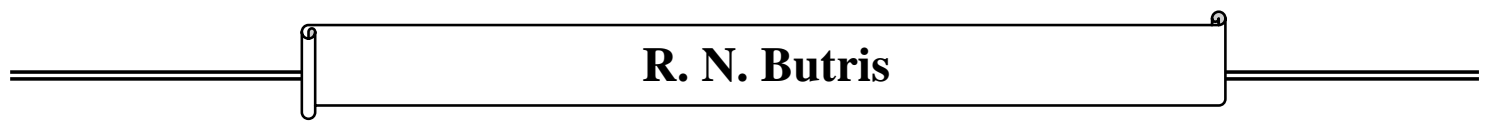

It follows form the inequality (19) that

$v_{m+1} \leq Q_{0} v_{m}$,

where $Q_{0}=\max _{t \in[0, T]} Q(t)$.

By iterating the inequality (21) gives

$v_{m+1} \leq Q_{0}^{m} v_{0}$,

which leads to the estimate

$\sum_{i=1}^{m} v_{i} \leq \sum_{i=1}^{m} Q_{0}^{i-1} v_{0}$.

Since the matrix $Q_{0}$ has eigen-values $q_{1}=0, q_{2}=0$ and $q_{3}=\left[\frac{T^{2}}{6}\left(K_{1}+K_{4} L_{1} T\right)+\frac{5 T}{6}\left(K_{2}+K_{4} L_{2} T\right)+2\left(K_{3}+K_{4} L_{3} T\right)\right]<1$, then the series (23) is uniformly convergent, i.e.

$\operatorname{Lim}_{m \rightarrow \infty} \sum_{i=1}^{m} Q_{0}^{i-1} v_{0}=\sum_{i=1}^{\infty} Q_{0}^{i-1} v_{0}=\left(E-Q_{0}\right)^{-1} v_{0}$.

The limiting relation (24) signifies a uniform convergent of the sequence $\left\{x_{m}\left(t, x_{0}\right), \dot{x}_{m}\left(t, x_{0}\right), \ddot{x}_{m}\left(t, x_{0}\right)\right\}$.

Let

$$
\begin{aligned}
& \operatorname{Lim}_{m \rightarrow \infty} x_{m}\left(t, x_{0}\right)=x_{\infty}\left(t, x_{0}\right), \\
& \operatorname{Lim}_{m \rightarrow \infty} \dot{x}_{m}\left(t, x_{0}\right)=\dot{x}_{\infty}\left(t, x_{0}\right),
\end{aligned}
$$

and

$$
\operatorname{Lim}_{m \rightarrow \infty} \dot{x}_{m}\left(t, x_{0}\right)=\dot{x}_{\infty}\left(t, x_{0}\right),
$$

By the inequality (21), the estimate (12) true for $m=1,2, \ldots$.

Thus $x_{\infty}\left(t, x_{0}\right)$ is the solution of the integral equation (11).

Finally, we have to show that $x\left(t, x_{0}\right)$ is unique solution of the system (1). On the contrary, we suppose that there is at least two different solutions $x\left(t, x_{0}\right)$ and $y\left(t, x_{0}\right)$ of (1).

From (11) the following identities are holds:

$$
\begin{aligned}
\left\|x\left(t, x_{0}\right)-y\left(t, x_{0}\right)\right\| \leq \alpha(t) \frac{T}{3}\left[\left(K_{1}+\right.\right. & \left.K_{4} L_{1} T\right)\left\|x\left(t, x_{0}\right)-y\left(t, x_{0}\right)\right\|+ \\
& +\left(K_{2}+K_{4} L_{2} T\right)\left\|\dot{x}\left(t, x_{0}\right)-\dot{y}\left(t, x_{0}\right)\right\|+ \\
& \left.+\left(K_{3}+K_{4} L_{3} T\right)\left\|\ddot{x}\left(t, x_{0}\right)-\ddot{y}\left(t, x_{0}\right)\right\|\right], \\
\left\|\dot{x}\left(t, x_{0}\right)-\dot{y}\left(t, x_{0}\right)\right\| \leq\left(\alpha(t)+\frac{T}{3}\right)[( & \left.K_{1}+K_{4} L_{1} T\right)\left\|x\left(t, x_{0}\right)-y\left(t, x_{0}\right)\right\|+ \\
+ & \left(K_{2}+K_{4} L_{2} T\right)\left\|\dot{x}\left(t, x_{0}\right)-\dot{y}\left(t, x_{0}\right)\right\|+ \\
+ & \left.\left(K_{3}+K_{4} L_{3} T\right)\left\|\ddot{x}\left(t, x_{0}\right)-\ddot{y}\left(t, x_{0}\right)\right\|\right],
\end{aligned}
$$


and so

$$
\begin{aligned}
\left\|\ddot{x}\left(t, x_{0}\right)-\ddot{y}\left(t, x_{0}\right)\right\| \leq 2\left[\left(K_{1}+K_{4} L_{1} T\right)\left\|x\left(t, x_{0}\right)-y\left(t, x_{0}\right)\right\|+\right. \\
+\left(K_{2}+K_{4} L_{2} T\right)\left\|\dot{x}\left(t, x_{0}\right)-\dot{y}\left(t, x_{0}\right)\right\|+ \\
\left.+\left(K_{3}+K_{4} L_{3} T\right)\left\|\ddot{x}_{m}\left(t, x_{0}\right)-\ddot{x}_{m-1}\left(t, x_{0}\right)\right\|\right] .
\end{aligned}
$$

thus

$$
\left(\begin{array}{l}
\left\|x\left(t, x_{0}\right)-y\left(t, x_{0}\right)\right\| \\
\left\|\dot{x}\left(t, x_{0}\right)-\dot{y}\left(t, x_{0}\right)\right\| \\
\left\|\ddot{x}\left(t, x_{0}\right)-\ddot{y}\left(t, x_{0}\right)\right\|
\end{array}\right) \leq Q_{0}\left(\begin{array}{l}
\left\|x\left(t, x_{0}\right)-y\left(t, x_{0}\right)\right\| \\
\left\|\dot{x}\left(t, x_{0}\right)-\dot{y}\left(t, x_{0}\right)\right\| \\
\left\|\ddot{x}\left(t, x_{0}\right)-\ddot{y}\left(t, x_{0}\right)\right\|
\end{array}\right) .
$$

By iterating whish we should find that

$$
\left(\begin{array}{l}
\left\|x\left(t, x_{0}\right)-y\left(t, x_{0}\right)\right\| \\
\left\|\dot{x}\left(t, x_{0}\right)-\dot{y}\left(t, x_{0}\right)\right\| \\
\left\|\ddot{x}\left(t, x_{0}\right)-\ddot{y}\left(t, x_{0}\right)\right\|
\end{array}\right) \leq Q_{0}^{m}\left(\begin{array}{l}
\left\|x\left(t, x_{0}\right)-y\left(t, x_{0}\right)\right\| \\
\left\|\dot{x}\left(t, x_{0}\right)-\dot{y}\left(t, x_{0}\right)\right\| \\
\left\|\ddot{x}\left(t, x_{0}\right)-\ddot{y}\left(t, x_{0}\right)\right\|
\end{array}\right)
$$

But $Q_{0}^{m} \rightarrow 0$ as $m \rightarrow \infty$, hence, proceeding in the last inequality to the limit we obtain that $x\left(t, x_{0}\right)=y\left(t, x_{0}\right), \dot{x}\left(t, x_{0}\right)=\dot{y}\left(t, x_{0}\right)$ and $\ddot{x}\left(t, x_{0}\right)=\ddot{y}\left(t, x_{0}\right)$ which proves the solution is unique, and this completes the proof of theorem 1 .

\section{Existence of Solution of (1)}

The problem of existence solution of the system (1) is uniquely connected with the existence of zeros of the function $\Delta\left(x_{0}\right)$, which has the form:-

$\Delta\left(x_{0}\right)=\frac{1}{T} \int_{0}^{T} L f\left(t, x_{\infty}\left(t, x_{0}\right), \dot{x}_{\infty}\left(t, x_{0}\right), \ddot{x}_{\infty}\left(t, x_{0}\right), \int_{t-T}^{t} g\left(s, x_{\infty}\left(s, x_{0}\right), \dot{x}_{\infty}\left(s, x_{0}\right), \ddot{x}_{\infty}\left(s, x_{0}\right)\right) d s\right) d t$

since this function is approximately determined from the sequence of functions:

$$
\begin{aligned}
& \Delta_{m}\left(x_{0}\right)=\frac{1}{T} \int_{0}^{T} L f\left(t, x_{m}\left(t, x_{0}\right), \dot{x}_{m}\left(t, x_{0}\right), \ddot{x}_{m}\left(t, x_{0}\right), \int_{t-T}^{t} g\left(s, x_{m}\left(s, x_{0}\right), \dot{x}_{m}\left(s, x_{0}\right), \ddot{x}_{m}\left(s, x_{0}\right)\right) d s\right) d t \\
& m=0,1,2, \ldots .
\end{aligned}
$$




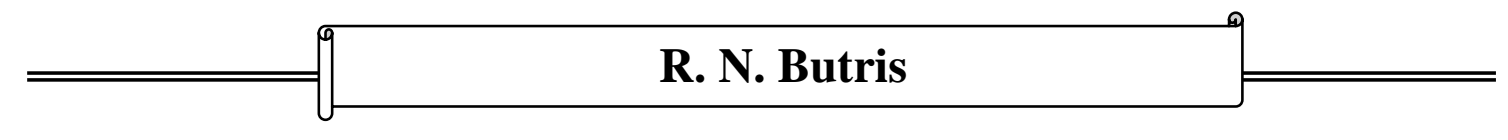

\section{Theorem 2:}

Let all assumptions and conditions of theorem 1 were given, then the following inequality:

$$
\left\|\Delta\left(x_{0}\right)-\Delta_{m}\left(x_{0}\right)\right\| \leq\left(\left(\begin{array}{c}
E_{1} \frac{T}{3} \\
E_{2} \frac{T}{3} \\
E_{3} \frac{T}{3}
\end{array}\right), Q_{0}^{m}(E-Q)^{-1} V_{0}\right)=\delta_{m}
$$

is holds for all $m \geq 1$ and $x_{0} \in D_{N}$.

\section{Proof:}

According to (26) and (27) we have

$$
\begin{aligned}
& \left\|\Delta\left(x_{0}\right)-\Delta_{m}\left(x_{0}\right)\right\| \leq \frac{1}{T} \int_{0}^{T} \alpha(t) \| f\left(t, x_{\infty}\left(t, x_{0}\right), \dot{x}_{\infty}\left(t, x_{0}\right), \ddot{x}_{\infty}\left(t, x_{0}\right), \int_{t-T}^{t} g\left(s, x_{\infty}\left(s, x_{0}\right), \dot{x}_{\infty}\left(s, x_{0}\right), \ddot{x}_{\infty}\left(s, x_{0}\right)\right) d s\right)- \\
& -f\left(t, x_{m}\left(t, x_{0}\right), \dot{x}_{m}\left(t, x_{0}\right), \ddot{x}_{m}\left(t, x_{0}\right), \int_{t-T}^{t} g\left(s, x_{m}\left(s, x_{0}\right), \dot{x}_{m}\left(s, x_{0}\right), \ddot{x}_{m}\left(s, x_{0}\right)\right) d s\right) \mid d t \\
& \leq \frac{T}{3}\left[K_{1}\left\|x_{\infty}\left(t, x_{0}\right)-x_{m}\left(t, x_{0}\right)\right\|+K_{2}\left\|\dot{x}_{\infty}\left(t, x_{0}\right)-\dot{x}_{m}\left(t, x_{0}\right)\right\|+\right. \\
& +K_{3}\left\|\ddot{x}_{\infty}\left(t, x_{0}\right)-\ddot{x}_{m}\left(t, x_{0}\right)\right\|+K_{4} L_{1}\left\|x_{\infty}\left(t, x_{0}\right)-x_{m}\left(t, x_{0}\right)\right\|+ \\
& \left.+K_{4} L_{2}\left\|\dot{x}_{\infty}\left(t, x_{0}\right)-\dot{x}_{m}\left(t, x_{0}\right)\right\|+K_{4} L_{3}\left\|\ddot{x}_{\infty}\left(t, x_{0}\right)-\ddot{x}_{m}\left(t, x_{0}\right)\right\|\right] \\
& =\frac{T}{3}\left[\left(K_{1}+K_{4} L_{1}\right)\left\|x_{\infty}\left(t, x_{0}\right)-x_{m}\left(t, x_{0}\right)\right\|+\right. \\
& +\left(K_{2}+K_{4} L_{2}\right)|| \dot{x}_{\infty}\left(t, x_{0}\right)-\dot{x}_{m}\left(t, x_{0}\right) \|+ \\
& \left.+\left(K_{3}+K_{4} L_{3}\right)\left\|\ddot{x}_{\infty}\left(t, x_{0}\right)-\ddot{x}_{m}\left(t, x_{0}\right)\right\|\right] \\
& \leq\left\langle\left(\begin{array}{l}
E_{1} \frac{T}{3} \\
E_{2} \frac{T}{3} \\
E_{3} \frac{T}{3}
\end{array}\right), Q_{0}^{m}(E-Q)^{-1} V_{0}\right)=\delta_{m},
\end{aligned}
$$

where $\langle$,$\rangle denotes the scalar product in the space R^{2}$ and $E_{1}=\left(K_{1}+K_{4} L_{1}\right), \quad E_{2}=\left(K_{2}+K_{4} L_{2}\right), \quad E_{3}=\left(K_{3}+K_{4} L_{3}\right), \quad \delta_{m}$ are a positive constants.

By using the inequality (28) we can prove the following theorem in a similar way to that of theorem 7.2 [1 ]. 


\section{Theorem 3:}

If the system of equations (1) satisfies the following conditions:

(a) the sequence of functions (26) has an isolated singular point $x_{0}=x_{\infty}$, $\Delta_{m}\left(x_{\infty}\right)=0$.

$\left(\mathbf{a}_{2}\right)$ the index of this point is nonzero.

$\left(\mathbf{a}_{3}\right)$ there exist a closed convex domain $D_{4}$ belonging to the domain $D_{N}$ and possessing a unique singular point $x_{\infty}$ such that on it's boundary $\Gamma_{D_{4}}$ the following inequality holds

$$
\min _{x_{0 \in \Gamma_{D_{4}}}}\left\|\Delta_{m}\left(x_{0}\right)\right\| \geq \delta_{m}
$$

for all $m \geq 1$. Then system (1) has a periodic solution $x=x(t)$ for which $x(0) \in D_{4}$.

\section{Remark 1: [2]}

When $R^{n}=R^{1}$, i.e. when $x$ is a scalar theorem 4 can be strengthens by giving up the requirement that the singular point should be isolated, thus we have.

\section{Theorem 4:}

Let the system of equations (1) be defined on the domain (2). Suppose that for $m \geq 0$, the function $\Delta_{m}\left(x_{0}\right)$ defined according to formula (27) satisfies the inequalities:

$$
\text { and } \left.\begin{array}{l}
\min \Delta_{m}\left(x_{0}\right) \leq-\delta_{m}, \\
\\
\max \Delta_{m}\left(x_{0}\right) \geq \delta_{m},
\end{array}\right]
$$

where $x_{0} \in[a+N, b-N]$. Then the system (1) has a periodic solution of period T, $x=x(t)$ for which $x(0) \in[a+N, b-N]$.

\section{Proof:}

Let $x_{1}$ and $x_{2}$ be any two points of the interval $[a+N, b-N]$ such that:

$\Delta_{m}\left(x_{1}\right)=\min \Delta_{m}(x)$, $\Delta_{m}\left(x_{2}\right)=\max \Delta_{m}(x)$,

where $x \in[a+N, b-N]$. Taking into account the inequalities (28) and (30), we have:

$$
\left.\begin{array}{l}
\Delta\left(x_{1}\right)=\Delta_{m}\left(x_{1}\right)+\left(\Delta\left(x_{1}\right)-\Delta_{m}\left(x_{1}\right)\right)<0, \\
\text { and } \\
\Delta\left(x_{2}\right)=\Delta_{m}\left(x_{2}\right)+\left(\Delta\left(x_{2}\right)-\Delta_{m}\left(x_{2}\right)\right)>0 .
\end{array}\right]
$$

It follows from (32) in virtue of the continuity of the $\Delta$-constant, that 


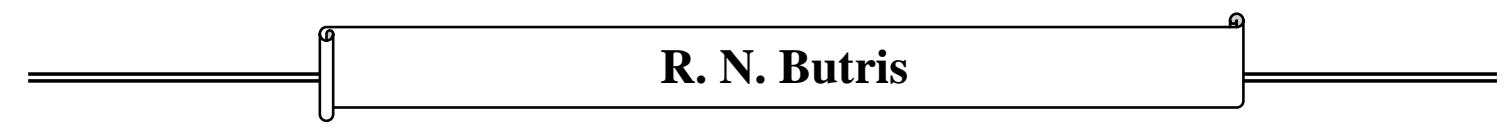

there exists a point $x_{\infty}, x_{\infty} \in\left[x_{1}, x_{2}\right]$, such that $\Delta\left(x_{\infty}\right)=0$. The last equality proves the theorem.

\section{Remark 2: [3]}

If the set $D_{f}$ dose not degenerate to a point, then the $\Delta$-constant of the system (37) may be considered as the function $\Delta=\Delta\left(0, x_{0}\right)$ given on the set $R^{1} \times D_{f}$. The properties are defined by:

\section{Theorem 5:}

Let

$\Delta: D_{N} \rightarrow R^{n}$, $\Delta\left(x_{0}\right)=\frac{1}{T} \int_{0}^{T} L f\left(t, x_{\infty}\left(t, x_{0}\right), \dot{x}_{\infty}\left(t, x_{0}\right), \ddot{x}_{\infty}\left(t, x_{0}\right), \int_{t-T}^{t} g\left(s, x_{\infty}\left(s, x_{0}\right), \dot{x}_{\infty}\left(s, x_{0}\right), \ddot{x}_{\infty}\left(s, x_{0}\right)\right) d s\right) d t$

where $x_{\infty}\left(t, x_{0}\right)$ is the limit of a sequence of periodic functions (9), then the following inequalities are holds:

$$
\left\|\Delta\left(x_{0}\right)\right\| \leq \frac{M T}{3}
$$

and

$$
\begin{aligned}
\left\|\Delta\left(x_{0}^{1}\right)-\Delta\left(x_{0}^{2}\right)\right\| \leq & E_{1}+\left(E_{1} E_{4}+E_{2} E_{4}+2 E_{3} E_{4}\left(1-2 E_{3}\right)^{-1}\left(E_{1}+E_{2} E_{4}\right)\right)\left(1-\frac{E_{1} T^{2}}{6}-\right. \\
& \left.\left.-\frac{E_{2} E_{4} T^{2}}{6}-\frac{E_{3} T^{2}}{3}\left(1-2 E_{3}\right)^{-1}\left(E_{1}+2 E_{2} E_{4}\right)\right)^{-1}\right]\left\|x_{0}^{1}-x_{0}^{2}\right\|,
\end{aligned}
$$

for all $x_{0}, x_{0}^{1}, x_{0}^{2} \in D_{N}$ and $E_{1}=\left(K_{1}+K_{4} L_{1}\right), E_{2}=\left(K_{2}+K_{4} L_{2}\right)$, $E_{3}=\left(K_{3}+K_{4} L_{3}\right)$ and

$$
E_{4}=\left(1-\frac{5 E_{2} T}{6}-\frac{5 E_{3} T}{3}\left(1-2 E_{3}\right)^{-1} E_{2}\right)^{-1}\left(\frac{5 E_{1} T}{6}-\frac{5 E_{3} T}{3}\left(1-2 E_{3}\right)^{-1} E_{1}\right) \text {. }
$$

\section{Proof:}

From the properties to the function $x_{\infty}\left(t, x_{0}\right)$ established by theorem 1 , it follows that the function $\Delta\left(x_{0}\right)$ is continuous and bounded in the domain $R^{1} \times D_{N}$. By using (33), we have:

$$
\left\|\Delta\left(x_{0}^{1}\right)-\Delta\left(x_{0}^{2}\right)\right\| \leq E_{1}\left\|x_{\infty}\left(t, x_{0}^{1}\right)-x_{\infty}\left(t, x_{0}^{2}\right)\right\|+E_{2}\left\|\dot{x}_{\infty}\left(t, x_{0}^{1}\right)-\dot{x}_{\infty}\left(t, x_{0}^{2}\right)\right\|+
$$




\section{Periodic solutions of second-order....}

$$
+E_{3}\left\|\ddot{x}_{\infty}\left(t, x_{0}^{1}\right)-\ddot{x}_{\infty}\left(t, x_{0}^{2}\right)\right\|
$$

where $x_{\infty}\left(t, x_{0}^{1}\right)$ and $x_{\infty}\left(t, x_{0}^{2}\right)$ are the solutions of the integral equation:

$$
x\left(t, x_{0}^{k}\right)=x_{0}^{k}+L^{2} f\left(t, x\left(t, x_{0}^{k}\right), \dot{x}\left(t, x_{0}^{k}\right), \ddot{x}\left(t, x_{0}^{k}\right), \int_{t-T}^{t} g\left(s, x\left(s, x_{0}^{k}\right), \dot{x}\left(s, x_{0}^{k}\right), \ddot{x}\left(s, x_{0}^{k}\right)\right) d s\right)
$$

where $\mathrm{k}=1,2$.

From (37), we find that

$$
\begin{aligned}
\left\|x_{\infty}\left(t, x_{0}^{1}\right)-x_{\infty}\left(t, x_{0}^{2}\right)\right\| & \leq\left\|x_{0}^{1}-x_{0}^{2}\right\|+\alpha(t)\left[E_{1}\left\|x_{\infty}\left(t, x_{0}^{1}\right)-x_{\infty}\left(t, x_{0}^{2}\right)\right\|+\right. \\
& \left.+E_{2}\left\|\dot{x}_{\infty}\left(t, x_{0}^{1}\right)-\dot{x}_{\infty}\left(t, x_{0}^{2}\right)\right\|+E_{3}\left\|\ddot{x}_{\infty}\left(t, x_{0}^{1}\right)-\ddot{x}_{\infty}\left(t, x_{0}^{2}\right)\right\|\right] \\
& \leq\left\|x_{0}^{1}-x_{0}^{2}\right\|+\frac{T^{2}}{6}\left[E_{1}\left\|x_{\infty}\left(t, x_{0}^{1}\right)-x_{\infty}\left(t, x_{0}^{2}\right)\right\|+\right. \\
& \left.+E_{2}\left\|\dot{x}_{\infty}\left(t, x_{0}^{1}\right)-\dot{x}_{\infty}\left(t, x_{0}^{2}\right)\right\|+E_{3}\left\|\ddot{x}_{\infty}\left(t, x_{0}^{1}\right)-\ddot{x}_{\infty}\left(t, x_{0}^{2}\right)\right\|\right]
\end{aligned}
$$

On differentiating $x_{\infty}\left(t, x_{0}^{1}\right)$ and $x_{\infty}\left(t, x_{0}^{2}\right)$, we get:

$$
\begin{array}{r}
\left\|\dot{x}_{\infty}\left(t, x_{0}^{1}\right)-\dot{x}_{\infty}\left(t, x_{0}^{2}\right)\right\| \leq \frac{5 T}{6}\left[E_{1}\left\|x_{\infty}\left(t, x_{0}^{1}\right)-x_{\infty}\left(t, x_{0}^{2}\right)\right\|+E_{2}\left\|\dot{x}_{\infty}\left(t, x_{0}^{1}\right)-\dot{x}_{\infty}\left(t, x_{0}^{2}\right)\right\|\right. \\
\left.+E_{3}\left\|\ddot{x}_{\infty}\left(t, x_{0}^{1}\right)-\ddot{x}_{\infty}\left(t, x_{0}^{2}\right)\right\|\right\rfloor \\
\ldots \ldots \text { (39) }
\end{array}
$$

Using the inequalities (37), (38) and (39) in (36) we have the inequality (35), and this proves the theorem.

\section{References}

1- Butris, R. N. "Existence of a solutions for a system of second order differential equations with boundary integral conditions", Iraq, Mosul, Educ. And Sci. J. Vol.18, pp.156-167, (1994).

2- Samoilenko A. M., Ronto N. I. "Numerical-analytic methods of investigating periodic solutions” Ukrain, Kive, (1976).

3- Mitropolsky Yu. A., Martynuk D. I., "Periodic solutions for oscillations systems with retarded argument” Ukrain, Kive, (1979). 